\title{
Um sistema de segurança elétrico hospitalar interligado por rede sem fio
}

\author{
Andréa Teresa R. Barbosa, Fábio Iaione e Rafael Fazolin Wendling
}

\begin{abstract}
Resumo- Este artigo apresenta um sistema de segurança elétrica (sistema IT-médico), composto por alguns equipamentos, que se comunicará por uma rede sem fio. $O$ objetivo foi desenvolver um dispositivo supervisor de isolação (DSI) e um anunciador com características próprias, que atendessem a norma técnica e apresentassem um menor custo para o hospital. Além disso, como nenhum outro sistema de segurança elétrica, encontrado comercialmente, possui comunicação sem fio entre seus componentes, o que proporciona facilidades estruturais e organizacionais para o Estabelecimento Assistencial de Saúde (EAS), realizou-se um estudo para definir a tecnologia wireless mais adequado a ser utilizada no sistema desenvolvido.
\end{abstract}

Palavras-Chave - Comunicação sem fio, Estabelecimento Assistencial de Saúde, Sistema de segurança elétrica, Sistema IT-médico.

Abstract - this work presents an electric safety system (IT system), composed by some equipments, that will communicate through wireless network. The objective was to develop an isolation monitoring device (IMD) and an announcer with own characteristics, that complied with the technical norm and that presented a small cost to the hospital. Besides, how neither electric safety system available commercially possesses wireless communication among yours components, which provides structural and organizational facility for the hospital, was realized study to define the better wireless technology to the developed system.

Index Terms - Wireless communication, hospital, electric safety system, IT system.

\section{INTRODUÇÃO}

Com a evolução e complexidade dos métodos e equipamentos inseridos nos estabelecimentos assistenciais de saúde (EAS), existe, nestes locais, uma necessidade cada vez maior de instalações elétrica seguras, que não causem riscos às pessoas envolvidas nos procedimentos médicos. Uma questão importante é que a falha de um dispositivo elétrico qualquer pode provocar risco de morte.

Diversas normas internacionais e nacionais abrangem os requisitos para instalações elétricas em EAS, mais especificamente nos setores de tratamentos críticos como Centros Cirúrgicos (CC) e Unidades de Tratamento Intensivo (UTI). As normas em ambientes de saúde visam, portanto, garantir o fornecimento seguro e confiável de energia aos

Andréa Teresa Riccio Barbosa e Fábio Iaione, Faculdade de Ciências Exatas e Tecnológica, Universidade Federal da Grande Dourados, Dourados, Brasil, e-mails: andreabarbosa@ufgd.edu.br, fabioiaione@ufgd.edu.br. Rafael Fazolin Wendling, Universidade Federal de Santa Catarina, Florianópolis, Brasil, e-mails: rfwedling@gmail.com.br. equipamentos eletromédicos (EEMs), proporcionando segurança aos pacientes, profissionais da área de saúde e visitantes.

Este artigo apresenta um sistema de segurança elétrica (sistema IT-médico) composto por um transformador isolador (TI), um dispositivo supervisor de isolação (DSI) e um anunciador.

O desenvolvimento deste conjunto de equipamentos foi motivado pela percepção dos problemas que ocorrem eventualmente durante procedimentos cirúrgicos, e pelos relatos encontrados nas literaturas das áreas da saúde sobre acidentes provocados por problemas de choque elétrico, durante cirurgias, nos pacientes e também na equipe médica [1]. Além disso, houve motivação pelo fato do hospital, em que foi testado o sistema, estar ampliando seu CC e UTI e, em consequiência, deveria implantar um sistema de segurança elétrica para atender a norma NBR 13534/95. Observa-se que a portaria do Ministério da Saúde n. ${ }^{\circ}$ 2662, de novembro de 95, e a RDC 50 da ANVISA exigem que quaisquer reformas em hospitais existentes ou construção de um novo hospital, siga a norma citada [3].

Um dos objetivos, portanto, foi desenvolver um DSI e um anunciador com características próprias, que atendessem a norma técnica e apresentassem um menor custo para o hospital.

Além disso, nenhum sistema de segurança elétrica, encontrado comercialmente, dispõe de comunicação sem fio que proporciona, neste caso, facilidades estruturais e organizacionais para o EAS. Portanto, realizou-se um estudo para definir a melhor tecnologia wireless a ser utilizada.

\section{MATERIAIS E MÉTODOS}

\section{A. Sistema IT-médico}

A utilização crescente de EEMs, na segunda metade do século XX, aumentou a preocupação com a prevenção de acidentes elétricos [4] [5]. Essa foi a principal idéia da norma IEC publicada como uma minuta, em 1976, pelo Comitê Técnico 62A, intitulada: "Common aspects of electrical equipment used in medical practice". Junto com inúmeras indicações sobre a instalação, essa minuta continha dois argumentos vitais para a instalação de sistemas de energia isolados (sistema IT-médico) em hospitais: aumento da confiabilidade do sistema de energia e redução das correntes de fuga [6].

A confiabilidade do sistema se refere à capacidade do fornecimento de energia sem interrupção, mesmo sob uma 
condição de falha de isolamento, como um curto-circuito faseterra, por exemplo. Isso é importante porque um EEM pode estar sendo usado para auxiliar ou substituir, temporariamente ou permanentemente, funções vitais do organismo.

A redução das correntes de fuga que passam pelo condutor de proteção é importante porque proporciona uma redução na "tensão de contato" do condutor de proteção (PE). A "tensão de contato" é gerada pelas correntes passando através da impedância de retorno do condutor de proteção [6]. Essa tensão, em um sistema TT sob uma falha de isolamento, é maior e se em contato com o corpo de um paciente, pode gerar uma corrente significativa através do mesmo. Cabe observar que a maioria dos EEMs, durante a utilização, está freqüentemente em contato com o paciente e/ou com os profissionais da área médica. Portanto, o perigo de choque elétrico sempre existe nesse ambiente [7]. Além disso, a diminuição da tensão de contato é importante devido a dois fatores (IEC TC 62A citada por [7]): incapacidade de o paciente detectar uma condição perigosa, devido ao seu estado e diminuição da resistência elétrica natural da pele, devido à inserção de um cateter, pelo tratamento da pele para fixação de um eletrodo ou em cirurgias cardíacas (tórax aberto), onde uma corrente elétrica pode provocar uma parada cardíaca.

\section{B. Monitoração do Sistema IT-médico}

Quando ocorre uma condição de falha em um sistema ITmédico, deve-se chamar atenção para o fato de que o sistema IT-médico pode transformar-se em um sistema convencional (TT), com um ponto de alimentação diretamente aterrado. Conseqüentemente, ele passa a apresentar todos os problemas e riscos associados ao sistema TT. Portanto, equipamentos foram desenvolvidos para monitorar o sistema IT-médico, sendo a utilização destes normalmente exigida por normas [6].

O primeiro é o DSI, que mede a resistência entre os condutores fase e o condutor de proteção, geralmente através da aplicação de uma tensão C.C., e sinaliza (som e luz) quando essa resistência decresce para $50 \mathrm{k} \Omega$ [6].

$\mathrm{O}$ segundo é o equipamento anunciador, que se localiza em uma das paredes do CC ou UTI, no posto de enfermagem central ou no setor de manutenção, e faz a sinalização sonora e visual dos eventos de alarme, e a identificação do DSI que está alarmando. Um anunciador pode comunicar-se com vários DSIs.

\section{Normas Técnicas}

No Brasil, em meados de maio de 1995, na tentativa de preencher a lacuna existente em termos de instalações elétricas hospitalares, foi aprovada a norma NBR 13534/95, elaborada pelo COBEI da ABNT. Essa norma exige uma série de requisitos para as instalações elétricas dos EAS [2].

Para tal, a norma classifica os recintos para fins médicos de acordo com dois critérios: o primeiro baseia-se no tipo de EEM presente no local durante o procedimento, e o segundo critério refere-se à segurança da alimentação com que os equipamentos do local devem ser providos.

Em relação ao primeiro critério, a norma define três grupos: grupo zero, que utiliza equipamento sem parte aplicada ao corpo humano; grupo um, com parte do EEM aplicada externamente ao corpo, ou parte aplicada a fluídos corporais, porém não aplicada ao coração; e grupo dois, com parte do equipamento aplicada ao coração, ou equipamentos essenciais à manutenção da vida.

$\mathrm{Na}$ alimentação elétrica em recintos do grupo dois, de acordo com a norma, cada cômodo ou grupo de cômodos deve prever um circuito de alimentação com Sistema IT (distribuição elétrica através de um transformador de isolação galvânica). O sistema IT-médico deve ser equipado com um DSI, dispondo de um sistema de alarme posicionado de tal forma que a instalação possa ser permanentemente supervisionada, durante sua utilização, pela equipe médica (lâmpada verde, amarela e alarme audível).

O DSI e o anunciador desenvolvidos fornecem segurança elétrica apropriada para recintos do grupo dois, como CC, UTIs, salas de cateterismo (hemodinâmica) e monitoração.

No sistema apresentado neste artigo, pretende-se que a comunicação, entre os dois dispositivos citados acima, passe a ser realizada através de uma rede sem fio. Para tal desenvolvimento, é necessário um estudo sobre emissão de radiofreqüência em ambientes hospitalares e tecnologias de redes sem fio.

\section{Emissão de radiofreqüência em ambientes hospitalares}

Todos os equipamentos eletrônicos são passíveis de sofrer interferências eletromagnéticas de outros equipamentos ou do ambiente onde estão em operação, assim como podem gerar ondas eletromagnéticos capazes de interferir no funcionamento de outros equipamentos.

Os efeitos dessas interferências são particularmente preocupantes quando ocorrem em EEM, sobretudo quando estão monitorando ou dando suporte à vida de um paciente [8].

As normas brasileiras e internacionais não contemplam nenhum tipo de abordagem específica sobre a emissão de ondas de rádio em ambiente hospitalar, apenas exigem que os equipamentos tenham proteção contra interferência eletromagnética e que essa emissão esteja dentro dos limites de compatibilidade eletromagnética.

A norma NBR/IEC 60601-1-2 [9] estabelece que os EEMs devam ser imunes aos campos elétricos de até $3 \mathrm{~V} / \mathrm{m}$, e que não devem emitir campos elétricos acima de $30 \mathrm{~dB} \mu \mathrm{V} / \mathrm{m}$ $(31,6 \mu \mathrm{V} / \mathrm{m})$ na faixa de $30 \mathrm{MHz}$ a $230 \mathrm{MHz}$, e de $37 \mathrm{~dB} \mu \mathrm{V} / \mathrm{m}$ $(70,6 \mu \mathrm{V} / \mathrm{m})$ na faixa de $230 \mathrm{MHz}$ a $1000 \mathrm{MHz}$, se o equipamento for classe B, isto é, para uso exclusivo em ambiente hospitalar.

Outra preocupação foi quanto à regulamentação da transmissão de informações por radiofrequiência na área médica, como será visto a seguir. 


\section{E. Regulamentação da comunicação por radiofreqüência na área médica}

O Federal Communications Commission (FCC) dos Estados Unidos, em junho de 2000, regulamentou as bandas de freqüência para a atuação dos sistemas de telemetria médica, instituindo a abreviatura WMTS (Wireless Medical Telemetry Service) para referenciar a categoria de serviços de telemetria médica sem fio.

Antes de junho de 2000, os sistemas WMTS atuavam em base secundária em antigas faixas de freqüência de TVs em VHF e UHF. Quando um serviço atua em base secundária sobre uma determinada faixa de freqüências, ele não deve causar interferências aos outros serviços (base primária) que também atuam nesta faixa. Estas faixas eram os canais 7 a 13 de VHF (174-216 MHz) e os canais 14 a 46 de UHF (470$668 \mathrm{MHz})$ [10].

Devido a problemas causados por outros equipamentos (transmissão de TV de alta definição), observou-se a necessidade de regulamentar faixa específica para os já existentes sistemas de WMTS. Definiram-se então, as faixas de 608-614 MHz (canal 37 de UHF), 1395-1400 MHz e 1429$1432 \mathrm{MHz}$ como frequiências de uso primário dos serviços de telemetria médica [10].

A regulamentação de junho de 2000 continuou permitindo o uso das bandas compartilhadas ISM (Instrumental, Scientific \& Medical) pelos sistemas WMTS nos Estados Unidos. Porém nestas bandas, os sistemas WMTS continuam atuando em base secundária e sem proteção legal contra danos causados por interferências dos serviços de base primária.

No Brasil, a ANATEL não especifica faixas exclusivas ou preferenciais para os sistemas de telemetria médica. No entanto, a ANATEL regulamenta nove bandas ISM [11], inclusive a banda ISM em 2,4 GHz, que é disponibilizada mundialmente e utilizada por tecnologias recentes de telecomunicações como o WiFi (Wireless Fidelity), Bluetooth, ZigBee, entre outros.

A despeito da alocação de bandas específicas para os WMTS, outras bandas de frequiência também são utilizadas através da operação dos sistemas de telemetria sobre infraestruturas de comunicação sem fio já existentes.

\section{F. Normas Associadas}

No Brasil existe uma legislação específica para regular os sistemas de espalhamento espectral. Esse conjunto de regras foi inicialmente definido na norma 002/93, aprovada pela portaria 046 do Ministério das Comunicações, de 2 de janeiro de 1993, modificado pela portaria 814, de 12 junho de 1996, e atualmente definido pelo Anexo à resolução 365 da ANATEL, emitido em 10 de maio de 2004 [12].

É importante salientar que no Brasil, como em vários outros países, a operação do sistema por espalhamento espectral não necessita de licença governamental para instalação e operação, desde que as regras e requisitos da Resolução 365 sejam obedecidos.

As faixas de frequiências estabelecidas para uso por equipamentos de radiocomunicação empregando a técnica de espalhamento espectral, para aplicações ponto a ponto e ponto multiponto, estão assim definidas: 902 a $928 \mathrm{MHz}, 2400$ a 2483,5 MHz e 5725 a $5850 \mathrm{MHz}$. A regulamentação vigente estabelece as condições de operações para os sistemas que operam por saltos de freqüência, para os sistemas que operam em seqüência direta e para os sistemas híbridos [12]:

1) Para sistemas de salto em freqüência (FHSS) operando nas faixas de $900 \mathrm{MHz}$, a potencia de pico máxima de saída do transmissor não deve ser superior a $1 \mathrm{~W}$, para sistemas que empreguem no mínimo 50 canais de salto, e $0,25 \mathrm{~W}$ para sistemas empregando menos de 50 canais de salto;

2) Sistemas FHSS operando nas faixas de $2,4 \mathrm{GHz}$ e $5,8 \mathrm{GHz}$ devem trabalhar com potência de pico máxima de saída do transmissor não superior a $1 \mathrm{~W}$;

3) Para sistemas de seqüência direta (DSSS) a potência de pico máxima de saída do transmissor não pode ser superior a $1 \mathrm{~W}$ para todas as faixas de freqüência.

\section{G. Tecnologias de redes sem fio}

O grupo de trabalho 11 do IEE, responsável pelos padrões IEEE 802.11 [12], considera a existência de quatro grandes grupos, em termos de redes sem fio:

1) WWAN - Wireless Wide Area Network: o grupo mais amplo em termos de alcance que é orientado para serviços de telecomunicações (voz e dados), em longas distâncias de transmissão;

2)WMAN - Wireless Metropolitan Area Network: neste grupo o objetivo é o acesso de banda larga em redes de âmbito metropolitano, cujo alcance é em torno de $6 \mathrm{~km}$;

3) WLAN - Wireless Local Area Network: tecnologias que se destinam a redes sem fio com alcance situado entre $100 \mathrm{e}$ $300 \mathrm{~m}$, freqüentemente como extensão ou alternativa a redes de cabeamento convencional (par de cobres, cabo coaxial ou fibra óptica);

4)WPAN - Wireless Personal Área Network: onde se enquadram tecnologias sem fio de pequeno alcance, entre 10 e $100 \mathrm{~m}$. Esta é a área de estudo e desenvolvimento normativo do grupo de trabalho 15 do IEEE, especializado nos padrões WPAN. Esta área compreende as redes sem fio que utilizam dispositivos como os computadores pessoais ou periféricos, sendo o campo de tecnologias como Bluetooth. O Low Rate WPAN, que tenta reunir uma baixa taxa de transferência de dados, baixa complexidade e oferecer um reduzido consumo energético, é parte integrante deste grupo e é sobre ele que foi desenvolvido o ZigBee.

A transmissão dos dados por radiofreqüência está, normalmente, associada ao empacotamento dos dados em frames que serão transmitidos segundo uma codificação. A codificação, o empacotamento e a transmissão dos dados, bem como o desempacotamento e o rastreamento do pacote são descritos segundo protocolos de comunicação.

Três dos protocolos de comunicação mais utilizados para comunicação de dados por radiofreqüência são: Wi-Fi, Bluetooth e Zigbee.

A tecnologia Bluetooth surgiu em 1999 e trouxe consigo um novo cenário de atuação de redes sem fio WPAN, já mencionada. Para a padronização deste novo conceito foi criado o grupo 802.15 da IEEE, sendo que o subgrupo 
802.15.1 ficou responsável pela regulamentação dessa tecnologia. Porém, para certas aplicações vislumbradas no cenário das WPANs, a tecnologia Bluetooth ainda fornece soluções superdimensionadas. Surgiu assim, o subgrupo IEEE 802.15.4 responsável pela regulamentação da tecnologia chamada ZigBee [13].

O subgrupo 802.15.4 está atualmente trabalhando em cooperação com a aliança de cinco empresas (Honeywell, Invensys, Mitsubishi, Motorola e Philips) que originalmente propôs a tecnologia ZigBee [13].

A tecnologia ZigBee atua nas faixas de ISM de $868 \mathrm{MHz}$, $910 \mathrm{MHz}$ e $2,4 \mathrm{GHz}$ com taxas de transmissão de no máximo $20 \mathrm{kbps}, 40 \mathrm{kbps}$ e $250 \mathrm{kbps}$ respectivamente, usando modulação de espalhamento espectral por seqüência direta (DSSS), que se constitui da multiplicação do sinal a ser transmitido por um sinal de "ruído". Este ruído é, na verdade, uma sequiência pseudo-aleatória gravada no transmissor e no receptor. A multiplicação do sinal no transmissor espalha o sinal pelo espectro, enquanto que uma segunda multiplicação no receptor reconstitui o sinal original [13].

Esta tecnologia foi desenvolvida para ter níveis muito baixos de consumo de energia, de forma a proporcionar autonomia de 6 meses a 2 anos para duas pilhas normais tamanho AA. Seu alcance pode variar entre 10 e $75 \mathrm{~m}$, dependendo dos limites de consumo de energia impostos por cada aplicação.

A pilha de protocolos utilizada pelo ZigBee é mais simples que os protocolos da tecnologia Bluetooth, tendo no máximo $32 \mathrm{kB}$ de código contra os $250 \mathrm{kB}$ do Bluetooth. Por ser mais simples, a tecnologia ZigBee poderá trabalhar com simples microcontroladores de 8 bits, como os da família MCS-51 [13].

A proposta da tecnologia ZigBee é fornecer soluções otimizadas para aplicações onde não se necessite toda a sofisticação da tecnologia Bluetooth, principalmente em aplicações com baixas taxas de transmissão de dados.

Devido às características apresentadas e como a quantidade de informações transmitidas do DSI para o anunciador é pequena (9600 bps no sistema por cabo), o ZigBee é a solução ideal para a transmissão sem fio.

\section{H. Algumas aplicações de redes sem fio na área médica}

O uso da tecnologia sem fio está presente em várias aplicações em ambientes médicos. Pode ser utilizada para concentrar informações dos pacientes em um único terminal, permitindo o monitoramento contínuo e mais eficiente por parte do corpo clínico, além de possibilitar, em alguns casos, uma maior mobilidade aos pacientes.

Além de hospitais e clínicas, a biotelemetria pode ser utilizada em ambulâncias na transmissão prévia dos sinais biológicos do socorrido ao hospital [14], de forma a possibilitar um pré-diagnóstico e uma melhor adequação da sala de emergência.

A biotelemetria é utilizada também no monitoramento de atletas em diversos esportes, como na Fórmula 1, na natação e no alpinismo. Pode ser usada também em residências, possibilitando a internação de pacientes em suas próprias casas, recebendo acompanhamento médico remotamente através de qualquer meio de comunicação disponível na residência, seja telefone [15], Internet, ou até mesmo redes de TV a cabo. Através destes meios, os sistemas de TeleHomeCare podem enviar sinais de alerta ou relatórios parciais às instituições de saúde [16].

O sistema proposto também fará uso da tecnologia sem fio em um EAS, para transmitir informações sobre segurança elétrica. Devido a isso, surgiu durante a pesquisa, a preocupação de se fazer uma avaliação do ambiente eletromagnético no centro cirúrgico do hospital que o sistema seria instalado.

\section{Avaliação do ambiente eletromagnético em um CC}

Para verificar o aspecto de compatibilidade eletromagnética no centro cirúrgico do hospital foram realizadas medições, pois é um setor de grande inserção tecnológica e onde será instalado o sistema de segurança desenvolvido.

As medições caracterizaram-se na parte radiada, pela medição dos campos elétricos e dos campos magnéticos, procurando contemplar, na medida do possível, uma ampla faixa de frequiências para verificação dos limites estabelecidos pelas normas de segurança.

Para medição de campos elétricos na faixa de $100 \mathrm{kHz}$ até $3 \mathrm{GHz}$ foi utilizado um sistema composto por um conjunto de antenas, um analisador de espectro portátil e um notebook.

Para medições no domínio da freqüência (principalmente na faixa de RF) a utilização de analisadores de espectro é considerada como a forma mais tradicional e eficiente para análise de sinais. Para a medição, o analisador de espectro empregado foi o FSH-3 (Rhode \& Schwartz) e o programa para controle de operação e armazenamento de dados foi o FSH View 8.0 [17].

Para medição de campos magnéticos na faixa de $1 \mathrm{~Hz}$ até $400 \mathrm{kHz}$ foi utilizado um medidor de campo magnético, ELT400 (Narda). Este sistema realiza medições em banda larga e não fornece uma análise espectral do sinal incidente, mas faz verificação direta da conformidade do campo medido [17].

$\mathrm{O}$ ensaio foi realizado no interior de uma sala do centro cirúrgico do hospital, com dimensões de $6,0 \mathrm{~m}$ x 6,18 m. A disposição de equipamentos e objetos no interior da sala avaliada obedece às recomendações do corpo clínico do hospital para os procedimentos de rotina. Esta sala estava equipada com uma unidade eletrocirúrgica (UEC), um carro de anestesia com ventilador pulmonar, um monitor multiparamétrico, uma serra elétrica de ossos, uma máquina de perfusão, uma bomba de infusão (tipo seringa) e um foco cirúrgico. Para as medições foi adotado o posicionamento cartesiano próximo dos equipamentos na sala cirúrgica. Outros critérios de melhor posicionamento são estabelecidos, como o trabalho de Valente e Raizer [18]

$\mathrm{O}$ resultado das medições de campo elétrico, com os EEMs em repouso, que avalia as emissões originadas por fontes eletromagnéticas situadas no ambiente externo é um espectro de frequiência típico de regiões urbanas. Ou seja, encontraram-se as rádios FM, com freqüência entre 98,6 e $103 \mathrm{MHz}$, com intensidade de campo de até $98,3 \mathrm{~dB} \mu \mathrm{V} / \mathrm{m}$; os 
canais de TV-VHF, 175 e $199 \mathrm{MHz}$, com intensidade de campo de até $87,99 \mathrm{~dB} \mu \mathrm{V} / \mathrm{m}$; os canais de TV-UHF, 472 e $496 \mathrm{MHz}$, com intensidade de campo de até 91,95 dB $\mu \mathrm{V} / \mathrm{m}$; serviços de comunicação, provavelmente walkie-talkies, 473 $\mathrm{MHz}$, com intensidade de campo de até $84,09 \mathrm{~dB} \mu \mathrm{V} / \mathrm{m}$; as bandas de telefonia celular A e B, $867 \mathrm{MHz}$, com intensidade de campo de até $98,04 \mathrm{~dB} \mu \mathrm{V} / \mathrm{m}$; e a banda C, 175 e 1880 $\mathrm{MHz}$, com intensidade de campo de até $85,36 \mathrm{~dB} \mu \mathrm{V} / \mathrm{m}$.

$\mathrm{O}$ resultado das medições com os EEMs em operação configura a situação do pior caso encontrado no ambiente avaliado. Pode-se notar que a operação dos EEMs contribui significativamente para o aumento do ruído espectral no ambiente. A situação mais crítica para este ambiente, dentro da faixa normativa, é observada para a freqüência de 103 $\mathrm{MHz}$, onde a intensidade do campo atinge a casa de 110,66 $\mathrm{dB} \mu \mathrm{V} / \mathrm{m}$. O maior valor de intensidade de campo observado foi devido a harmônicos da UEC em $962 \mathrm{kHz}$ com valores de $118,45 \mathrm{~dB} \mu \mathrm{V} / \mathrm{m}$.

Os valores de campo magnético no ponto próximo à região de operação da unidade eletrocirúrgica, pior caso, atingem $12,33 \%$ do limite estipulado pela ICNIRP para exposição de público geral.

Colocando-se o DSI desenvolvido no centro cirúrgico, o resultado da medição de campo elétrico de $100 \mathrm{kHz}$ a $3 \mathrm{GHz}$, durante sua operação, não apresentou grande contribuição eletromagnética ao ambiente.

Para o caso do hospital em estudo, portanto, o risco de emissão eletromagnética na configuração utilizada durante os ensaios tem se mostrado baixo, uma vez que o ambiente é propício à operação segura dos EEMs e do DSI e não apresenta evidências críticas de emissões radiadas.

\section{J. Sistema de segurança elétrica desenvolvido}

A etapa inicial do processo de desenvolvimento do sistema de segurança elétrica foi o planejamento, que incluiu: análise dos produtos existentes no mercado, estudo de viabilidade técnica e especificação do sistema.

O DSI projetado e desenvolvido é um equipamento microcontrolado que tem a função de medir, mostrar e monitorar a resistência elétrica (indicada em quilohms - $\mathrm{k} \Omega$ ) entre as saídas de um transformador isolador $(220 \mathrm{v} / 220 \mathrm{v}$ ou $110 \mathrm{v} / 110 \mathrm{v})$ e o terra de proteção, e também, a temperatura (indicada em graus centígrados $-{ }^{\circ} \mathrm{C}$ ) e a corrente (indicada em Ampères - A) de operação desse transformador.

O transformador isolador foi adquirido de um fabricante nacional e é usado para alimentar a sala cirúrgica, UTI ou outro ambiente hospitalar que necessite de proteção contra choques elétricos.

Os valores de alarme para a resistência de isolação, temperatura e corrente são ajustados no próprio DSI através de teclas e do visor LCD, permitindo assim a sinalização de eventos como baixa resistência de isolação, sobretemperatura e sobrecorrente. A resistência de isolação é medida através da aplicação de uma tensão CC de $12 \mathrm{~V}$ entre o terra de proteção e uma das saídas do transformador isolador.

Vários DSIs poderão ser conectados em uma pequena rede sem fio, onde existe um equipamento anunciador que fará a sinalização sonora e visual dos eventos de alarme, e a identificação do DSI que está alarmando. O anunciador é alimentado por uma fonte de 12 VCC (wall cube) e pode ser conectado em até 10 DSIs. Além disso, ele registra os eventos (falhas de segurança elétrica) em uma memória EEPROM interna, proporcionando assim um histórico que poderá ser verificado conectando-se um computador (via porta serial) e utilizando-se um aplicativo do tipo terminal de texto, como o HyperTerminal do Windows.

As conexões (terminais) existentes no DSI são: terminais para alimentação com CA $(14+14 \mathrm{~V}$ e $4+4 \mathrm{~V})$, terminais para conexão de um sensor de temperatura, terminais para conexão de um termostato, terminais para conexão de um transformador de corrente, terminais para conexão no terra de proteção, terminais para conexão em uma das saídas do transformador isolador e um conector (9 pinos) para conexão com a porta serial de um computador IBM-PC e para gravação do firmware no microcontrolador. A alimentação do DSI é proporcionada por uma fonte externa, através de 6 cabos.

Além dos alarmes citados, relacionados às falhas na segurança elétrica, o DSI também detecta e sinaliza falhas nele próprio, tais como, rompimento de um dos cabos de conexão ao terra de proteção, rompimento de um dos cabos de conexão à saída do transformador isolador, rompimento nos cabos do termostato, rompimento ou curto-circuito nos cabos do sensor de temperatura e rompimento ou curto-circuito nos cabos do transformador de corrente.

Para utilizar o DSI e obter as informações necessárias no anunciador, alguns parâmetros deverão ser ajustados, tais como: alarme de temperatura do transformador isolador, alarme de corrente de carga do transformador isolador, alarme de resistência de fuga do sistema IT, configuração do DSI na rede, hora e data do anunciador, número de leitos ligados ao IT/DSI. Estes parâmetros são facilmente ajustados no teclado de membrana conforme instruções que aparecem no visor.

A comunicação sem fio entre os DSIs e o anunciador terá topologia tipo estrela (piconet mestre-escravo), pois possui um dispositivo coordenador (anunciador) que gerenciará a comunicação entre os dispositivos (DSIs). O coordenador, portanto, se encarregará de toda a comunicação em um dado canal de rádio e, em sua área de alcance no hospital (personal area network - PAN), será capaz de se comunicar com qualquer DSI. O anunciador será classificado como um dispositivo de função completa (full function devices - FFD) e estará necessariamente no modo de recepção sempre que não estiver transmitindo. Os DSIs serão classificados como dispositivos de função reduzida (reduced function device RFD). Eles não participarão de qualquer atividade de roteamento e se comunicarão somente com o dispositivo FFD. Não assumirão, portanto, qualquer função de coordenação de rede.

No anunciador serão realizados os ajustes de parâmetros da rede, e ele fará o gerenciamento, o armazenamento e a distribuição das mensagens entre nós de rede, transmitindo 
informações pela rede e operando tipicamente em estado ativo.

Os DSIs terão função passiva na rede, efetuando buscas por redes disponíveis, fazendo transferência dos dados de aplicação, determinando o status dos dados, solicitando dados ao coordenador de rede e poderão permanecer no estado sleep por longos períodos.

O sistema, inicialmente utilizando comunicação por fio, foi instalado no departamento de pesquisa do hospital, onde permaneceu em teste por aproximadamente 3 meses. Posteriormente, foi instalado para monitorar um leito da UTI pediátrica. Durante a utilização, ocorreu um evento de sinalização (sonora e escrita no visor) do anunciador. Constatou-se que ocorreu uma queda na resistência de isolamento do sistema IT, e uma verificação nos equipamentos alimentados por esse sistema revelou uma "caixa de tomadas" com um cabo rompido provocando um curto-circuito entre uma das fases e o terra. Nota-se que sem o uso deste sistema de segurança que alarma quando há problemas, o corpo clínico não seria informado do ocorrido e, caso fosse internado algum paciente no leito que é alimentado com o sistema elétrico avariado, este poderia sofrer choques elétricos.

\section{CONCLUSÃO}

Foi realizado um estudo para verificar a tecnologia de comunicação sem fio mais indicada para o aperfeiçoamento do sistema IT-médico. Essa melhoria substituirá os cabos para comunicação de dados entre os equipamentos por comunicação sem fio. Houve um estudo mais aprofundado e uma indicação para o uso da tecnologia ZigBee. Observa-se, porém que poderia ser utilizado o sistema Bluetooth e Wi-fi, pois todas estas tecnologias operam na freqüência de $2,4 \mathrm{GHz}$ e não necessitam de nenhum tipo especial de homologação para seu uso, pois operam na faixa ISM. A tecnologia ZigBee possui um menor consumo de energia, pilhas de protocolos mais simples e menores, exigindo menos processamento por parte do controlador.

Verificou-se que não existe nenhuma restrição específica estabelecida por norma sobre a emissão de radiofrequiência para comunicação de dados no ambiente hospitalar. Também se constatou que as emissões eletromagnéticas já existentes no ambiente hospitalar não interferirão na tecnologia proposta de comunicação sem fio.

A comunicação sem fio é interessante pela flexibilidade que proporciona, pois, dentro da área de cobertura, a localização do anunciador poderá ser definida conforme a necessidade do momento e exigirá apenas a presença de uma tomada elétrica nas proximidades. Além disso, permite que a rede alcance facilmente lugares onde os cabos de comunicação exigiriam modificações prediais para chegarem. Também, há facilidade na instalação que pode ser rápida, evitando a passagem de cabos através de paredes, canaletas e forros, proporcionando assim o uso mais eficiente do espaço físico. Outra vantagem é que diversas topologias podem ser configuradas para atender aplicações específicas. As configurações são facilmente alteradas e há facilidade de expansão e manutenção reduzida.

Como as normas exigem que o sistema IT-médico seja instalado apenas em EASs em construção ou em reforma, devido à necessidade de instalações adequadas (quadros de distribuição, eletrodutos e cabeamento), percebe-se que a utilização de comunicação sem fio facilitará a instalação desses sistemas de segurança elétrica em qualquer EAS. Em relação às limitações, acredita-se que a principal seja a interferência de outras tecnologias operando na mesma freqüência, utilizadas futuramente no mesmo ambiente.

Espera-se que esse trabalho dê subsídios para implementação de comunicação wireless no sistema de segurança elétrica desenvolvido.

\section{REFERENCIAS}

[1] Webster, J.G. Medical instrumentation: application and design. Boston: editor, 1978.

[2] Associação Brasileira de Normas Técnicas. Instalações elétricas em estabelecimentos assistenciais de saúde- Requisitos para segurança, NBR 13534. Rio de Janeiro, 1995.

[3] Agência Nacional de Vigilância Sanitária. Resolução - RDC n ${ }^{\circ} 50$. Dispõe sobre o Regulamento Técnico para planejamento, programação, elaboração e avaliação de projetos físicos de estabelecimentos assistenciais de saúde, 2002

[4] Naseerudin, S.M. "Electrical Safety in Healthcare Facilities." IEP-SAC Journal. Saudi Arabia, p. 99-103, 2004.

[5] Atkin, D. H.; Orkin, L.R. (1973) "Electrocution in the Operating Room". Anesthesiology. v. 38, n. 2, p. 181-183, 1973.

[6] Hofheinz, W. Electrical Safety - Ungrounded Power Supply Systems (ITSystems) in Medical Sites. WGKT, Gruenberg, Germany, 2003.

[7] IEC TC 62

[8] Cabral, S.B.C.; Mühlen, S. S. Interferência Eletromagnética no Ambiente Hospitalar. Multiciência, 2005.

[9] IEEE 802.15.4. The Institute of Electrical and Electronic Engineers, Standard Report: Wireless Medium Access Control (MAC) and Physical Layer (PHY) Specification for Low-Rate Wireless Personal Area Network (LRWPANs), New York, EUA, 2007.

[10] Barbosa, A. T. R, Relatório Técnico final de pós-doutorado, 2007.

[11] USA. Amendment of Parts 2 and 95 of the Commission's Rules to Create a wireless medical Telemetry Service. Federal Communications Commission, Washington, Jun. 2000. Disponível em: http://www.fcc.gov/Document_Indexes/ Engineering_Technology/2000_index_OET_Order.html

[12] ANATEL. Regulamento sobre equipamentos de radiocomunicação de radiação restrita. Brasília: s. n., anexo a Resolução 365, 19 de maio de 2004.

[13] Zigbee Alliance. Fevereiro de 2007. http://www.zigbee.com.

[14] Cullen, J.; Gaasch, W. R.; Gagliano, D. M.; Unawardane, R.; Hu, P. F.;Lamonte, M. P.; Mackenzie, C. F.; Xiao, Y.; Mobile telemedicine system. In: Annual International Conference Of The Ieee Engineering In Medicine And Biology Society. Proceedings. Atlanta. v. 2, p. 707, 1999.

[15] Orlov, O. I.; Drozdov, D. V.; Doarn, C. R.; Merrell, R. C.; Wireless ECG monitoring by telephone. Telemedicine Journal and e-Health. Larchmont, v. 7, n. 1, p. 33-38, 2001.

[16] LEE, H.; PARK, D. Bio-medical FM-FM-FSK radiotelemetry system for multi-signal transmission. In: Annual International Conference Of The IEEE Engineering In Medicine And Biology Society. Proceedings. Chicago. v. 2, p. 1553-1555, 2000

[17] Relatório Técnico de medidas $n^{\circ} 01 / 2007$, Universidade Federal do Rio Grande do Sul, Escola de Engenharia, Porto Alegre, 2007.

[18] VALENTE JR., W.; RAIZER, A. Avaliação do ambiente eletromagnético em estabelecimento assistenciais de saúde. Revista Brasileira de Engenharia Biomédica, v. 24, n.3, p. 215-217, 2008. 